\title{
The dust-enshrouded microquasar candidate AX J1639.0-4642 = IGR J16393-4643
}

\author{
J. A. Combi ${ }^{1,2,3}$, M. Ribó ${ }^{2}$, I. F. Mirabel ${ }^{2,4}$, and M. Sugizaki ${ }^{5}$ \\ ${ }^{1}$ Departamento de Física, Escuela Politécnica Superior, Univ. de Jaén, Virgen de la Cabeza 2, 23071 Jaén, Spain \\ e-mail: jcombi@ujaen.es \\ 2 Service d'Astrophysique, CEA Saclay, Bât. 709, L'Orme des Merisiers, 91191 Gif-sur-Yvette Cedex, France \\ e-mail: [mribo;mirabel]@discovery.saclay.cea.fr \\ 3 Instituto Argentino de Radioastronomía, C.C.5, (1894) Villa Elisa, Buenos Aires, Argentina \\ ${ }^{4}$ Instituto de Astronomía y Física del Espacio, CONICET, C.C.67, Suc. 28, 1428 Buenos Aires, Argentina \\ 5 Santa Cruz Institute for Particle Physics, University of California, 1156 High Street, Santa Cruz, CA 95064, USA \\ e-mail: sugizaki@scipp.ucsc.edu
}

Received 31 January 2004 / Accepted 17 April 2004

\begin{abstract}
We present a multiwavelength study of the field containing the unidentified X-ray source AX J1639.0-4642, discovered with the ASCA observatory and recently detected with the IBIS telescope, onboard the INTEGRAL satellite, dubbed IGR J16393-4643. The huge hydrogen column density towards the source, the hard spectral index in the $0.7-10 \mathrm{keV}$ band and its flux variability suggest that the source is a High Mass X-ray Binary (HMXB) enshrouded by dust. Our search reveals the presence of a non-thermal radio counterpart within the X-ray error box. After a study of the broadband emission from X-rays to the radio domain, we propose that AX J1639.0-4642 is a dust-enshrouded Microquasar (MQ) candidate. In addition, the $\mathrm{X}$-ray source is well within the $95 \%$ location contour of the unidentified $\gamma$-ray source 3EG J1639-4702. The main properties of AX J1639.0-4642/3EG J1639-4702 are consistent with those of two other MQs previously proposed to display high-energy $\gamma$-ray emission.
\end{abstract}

Key words. stars: general - stars: binaries: general - radio continuum: stars - gamma rays: observations infrared: stars

\section{Introduction}

Microquasars are X-ray binary systems with collimated relativistic jets produced by the accretion of matter from a normal star to a compact object such as a neutron star or a black hole (Mirabel \& Rodríguez 1999; Fender 2004). This highly energetic process has observable consequences from radio to hard X-ray and possibly up to $\gamma$-ray energies (Paredes et al. 2000). An interesting property of microquasars is that, contrary to what happens in quasars, they offer a unique opportunity to study accretion/ejection and related phenomena on human timescales (Mirabel \& Rodríguez 1999). Although the total number of known MQ sources in the Galaxy is around 16 (Ribó 2003), this number could increase up to $\sim 40$ if, as has been suggested by Fender \& Hendry (2000), all Radio Emitting X-ray Binaries (REXBs), are MQs. In any case, it is desirable to enlarge the MQ sample to allow meaningful statistical studies. To this end, Paredes et al. (2002) have recently carried out a search for new REXBs at galactic latitudes of $|b|<5^{\circ}$, and although the first results were promising (Ribó et al. 2002a), recent optical spectroscopic observations (Martí et al. 2004) reveal that most of the studied sources, if not all, are extragalactic quasars.

On the other hand, with the advent of the X-ray/ $\gamma$-ray INTEGRAL satellite the possibility of finding new MQ candidates has increased. During the last months, a few highly absorbed hard X-ray sources have been discovered with the IBIS detector in the galactic plane towards the Norma spiral arm (e.g. IGR J16318-4848, Revnivtsev et al. 2003; IGR J16320-4751, Rodriguez et al. 2003). These sources present an unusually high intrinsic hydrogen column density up to $2 \times 10^{24} \mathrm{~cm}^{-2}$, revealing a population of dust-enshrouded hard X-ray sources that are difficult to detect below $5 \mathrm{keV}$. Although at present the nature of these sources remains unclear, it is believed that they could be high mass X-ray binary systems (Revnivtsev 2003), and eventually dust-enshrouded MQs.

With the aim of discovering new MQs we have focused our attention on several yet unexplored potential systems. In this paper, we present an in-depth study of the unidentified X-ray source AX J1639.0-4642, which was discovered with ASCA (Sugizaki et al. 2001), and show that it may be a MQ. We present a re-analysis of the ASCA data in Sect. 2, we describe our multiwavelength approach in Sect. 3, we discuss on the 
possible origin of the detected broadband emission in Sect. 4 and we state our conclusions in Sect. 5.

\section{Re-analysis of the ASCA data of AX J1639.0-4642}

The unidentified X-ray source AX J1639.0-4642 was discovered by the Advanced Satellite for Cosmology and Astrophysics (ASCA) observatory during a survey of the central region of the galactic plane, performed in the $0.7-10 \mathrm{keV}$ energy range (Sugizaki et al. 2001). The source is superimposed on the galactic Norma spiral arm, and located at $(l, b)=$ $\left(337^{\circ} .993,+0^{\circ} .072\right),(\alpha, \delta)_{\mathrm{J} 2000.0}=\left(16^{\mathrm{h}} 39^{\mathrm{m}} 04^{\mathrm{s}} .3,-46^{\circ} 42^{\prime} 47^{\prime \prime}\right)$ $\left(90 \%\right.$ or $1.6 \sigma$ uncertainty of $\left.1^{\prime}\right)$. It presented a flux of $F_{\mathrm{X}(0.7-10 \mathrm{keV})}=(19.2 \pm 2.2) \times 10^{-12} \mathrm{erg} \mathrm{cm}^{-2} \mathrm{~s}^{-1}$ (and variable X-ray emission with a confidence $\geq 99 \%$ ). Its spectrum was fit with a power-law with a very hard photon index $\Gamma=$ $-0.01_{-0.60}^{+0.66}$ and a poorly constrained hydrogen column density of $N_{\mathrm{H}}=12.82_{-6.88}^{+8.58} \times 10^{22} \mathrm{~cm}^{-2}$. The source has recently been detected by the IBIS telescope onboard the INTEGRAL satellite (Malizia et al. 2004).

\subsection{Observations and data reduction}

To investigate in more detail the properties of this X-ray source, we have re-analyzed the original ASCA data. The observations were performed on 1997 September 10, with two different pointings in the overlapped region of successive GIS fields of view. The first pointed observation (OBS\#1) started on 07:01:34 (UT) and had an exposure time of $11 \mathrm{ks}$, while the second one (OBS\#2) started on 12:41:50 (UT) and lasted $6 \mathrm{ks}$. The source was located at an off-axis angle of $20^{\prime}$ and $14^{\prime}$ from the optical axis of the X-ray telescope in OBS\#1 and OBS\#2, respectively.

The data reduction and the extraction of events was performed based on the standard procedure, as described in Sugizaki et al. (2001). The source events were extracted from a circular region of $3 \mathrm{~mm}$ radius on the GIS detector around the source position. Since the background is angle-dependent, it was collected from a source-free region with the same offaxis angle as the source. The data of the GIS-2 and the GIS-3 were summed to improve photon statistics.

\subsection{Lightcurve}

To examine the time variability of AX J1639.0-4642, we extracted lightcurves and fitted them with a constant model. We show in Fig. 1 the lightcurves obtained throughout the two pointed observations in the total energy band $0.7-10 \mathrm{keV}$, and in the energy bands of $0.7-5$ and $5-10 \mathrm{keV}$. The best-fit constant model and the reduced chi-squared $\left(\chi_{v}^{2}\right)$ of each lightcurve are also shown in Fig. 1.

In the $0.7-5 \mathrm{keV}$ range, the values of $\chi_{v}^{2}$ are within the $90 \%$ confidence limits, thus the flux can be considered to be constant. However, in the 5-10 keV range, the $\chi_{v}^{2}$ values exceed the $99 \%$ confidence limits, which implies that the flux is significantly variable. Nevertheless, the photon statistics are not

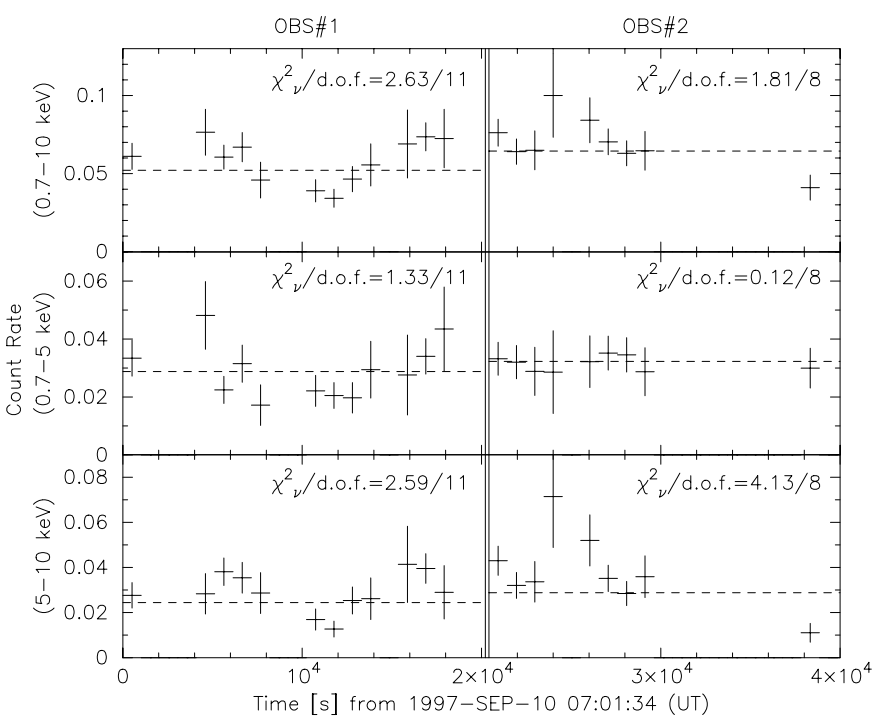

Fig. 1. Background-subtracted GIS lightcurves of AX J1639.0-4642 in $0.7-10 \mathrm{keV}$ (top), $0.7-5 \mathrm{keV}$ (middle) and 5-10 keV (bottom) throughout OBS\#1 $\left(0-2 \times 10^{4} \mathrm{~s}\right)$ and OBS\#2 $\left(2-4 \times 10^{4} \mathrm{~s}\right)$. Error bars represent $1 \sigma$ statistical uncertainty. Dashed lines represent the bestfit constant models. Reduced chi-squared $\left(\chi_{v}^{2}\right)$ and degree of freedom (d.o.f.) of the best-fit models are shown together. The different average count rates between OBS\#1 and OBS\#2 are explained by the change of the source position on the detector.

good enough to conclude that the source has larger variability in the hard band than in the soft band.

\subsection{Spectral analysis}

The spectral parameters of AX J1639.0-4642 in the ASCA galactic faint source survey in Sugizaki et al. (2001) were based on the OBS\#1 data. However, in OBS\#1 the source was observed at a large off-axis angle of $20^{\prime}$, where the response function of the X-ray telescope is not well calibrated. Therefore, we have re-analyzed the X-ray spectrum using the OBS\#2 data, when the source was observed with a lower off-axis angle of $14^{\prime}$. All spectral uncertainties represent $90 \%$ confidence limits, hereafter. We show in Fig. 2 the obtained X-ray spectrum in OBS\#2. We first fitted the spectrum with a power-law model with an absorption of neutral matter of solar abundance. The obtained best-fit parameters were photon index, $\Gamma=-0.28_{-0.56}^{+1.1}$, absorption hydrogen column density, $N_{\mathrm{H}}=11_{-7}^{+17} \times 10^{22} \mathrm{~cm}^{-2}$, and flux in the $0.7-10 \mathrm{keV}$ band, $F_{0.7-10 \mathrm{keV}}=(11 \pm 1.2) \times$ $10^{-12} \mathrm{erg} \mathrm{cm}^{-2} \mathrm{~s}^{-1}$. These values are consistent with those from OBS\#1 in the ASCA galactic faint X-ray catalog, although the flux is somehow smaller. The obtained value for $\Gamma \sim 0$ is very poorly constrained, and is certainly smaller than the typical values of $\Gamma>1$ found in other X-ray binaries. Therefore, to estimate a more realistic absorption column density, we next fitted the spectrum with the power-law model fixing the photon index $\Gamma$ to be 1 , which is typical of hard X-ray sources such as HMXBs. The obtained acceptable range of $N_{\mathrm{H}}$ with $90 \%$ confidence was $30_{-9}^{+12} \times 10^{22} \mathrm{~cm}^{-2}$. We also attempted to make a fit with a blackbody as an example of thermal spectral models, and derived a temperature of $k T=5.5_{-2.7}^{+40} \mathrm{keV}$ as the 


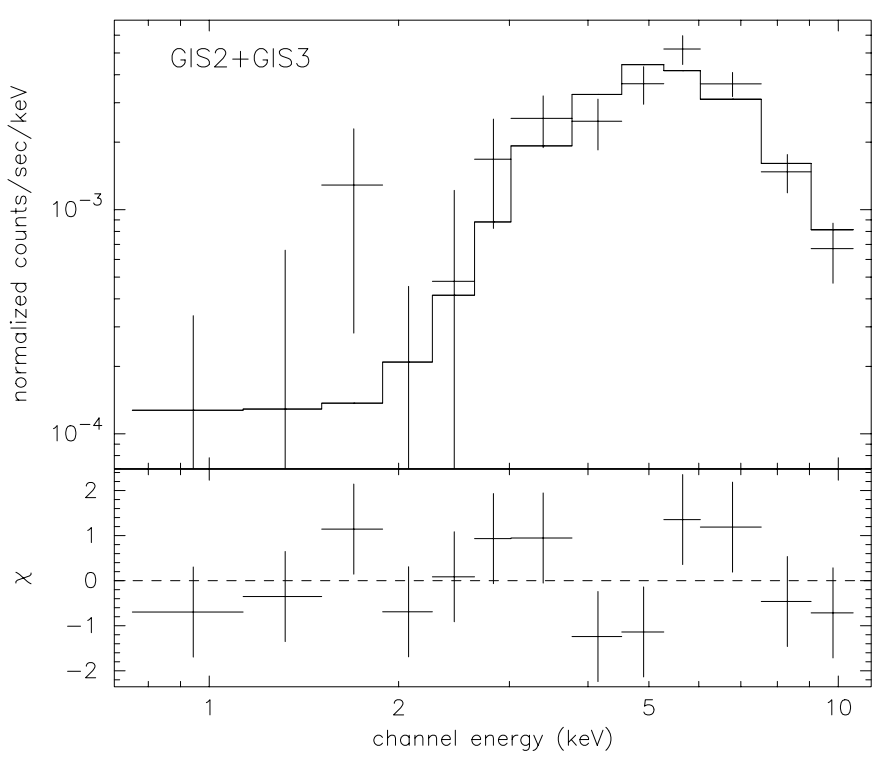

Fig. 2. GIS spectrum of AX J1639.0-4642 with the best-fit powerlaw model with an absorption of neutral matter with solar abundance (top), and the residuals (bottom). Error bars represent $1 \sigma$ statistical uncertainty.

Table 1. Summary of spectral fitting results. All errors represent the $90 \%$ confidence limits of statistical uncertainty.

\begin{tabular}{lccc}
\hline \hline & & Model \\
Parameter & PL & PL $(\Gamma=1$ :fixed $)$ & BB \\
\hline$\Gamma$ or $k T^{a}$ & $-0.28_{-0.56}^{+1.1}$ & $1:$ fix & $5.5_{-2.7}^{+40}$ \\
$N_{\mathrm{H}}{ }^{b}$ & $11_{-7}^{+17}$ & $30_{-9}^{+12}$ & $10_{-6}^{+13}$ \\
$F_{0.7-10 \mathrm{keV}^{c}}$ & $1.1 \pm 0.13$ & $0.99 \pm 0.12$ & $1.1 \pm 0.13$ \\
\hline$\chi_{v}^{2} /$ d.o.f. & $1.10 / 10$ & $1.33 / 11$ & $1.04 / 10$ \\
\hline
\end{tabular}

${ }^{a} k T$ in units of keV. ${ }^{b}$ Absorption hydrogen column density in units of $10^{22} \mathrm{~cm}^{-2}$. ${ }^{c}$ Flux in units of $10^{-11} \mathrm{erg} \mathrm{cm}^{-2} \mathrm{~s}^{-1}$.

best fit. However, the thermal spectral model is unlikely because the source has been detected by INTEGRAL ISGRI in the $15-40 \mathrm{keV}$ band. Unfortunately, the photon statistics of the ASCA observations are not good enough to exclude any model with a confidence greater than $99 \%$. The results of the spectral fitting are summarized in Table 1.

\section{Multiwavelength study of the AX J1639.0-4642 field}

With the aim of finding any possible radio counterpart within the location error box of the X-ray source, we have used the Molonglo Galactic Plane Survey (MGPS) conducted at $843 \mathrm{MHz}$ with the Molonglo Observatory Synthesis Telescope (MOST) (Green et al. 1999). Observations of the region of interest were conducted on 1990 June 9 and on 1992 April 16. We show in Fig. 3 the MOST contour image of the surroundings of AX J1639.0-4642 obtained during the second run. A bright radio source (hereafter MOST J1639.0-4642) can be clearly seen well within the error box of the X-ray source. Although the source appears somewhat extended, it

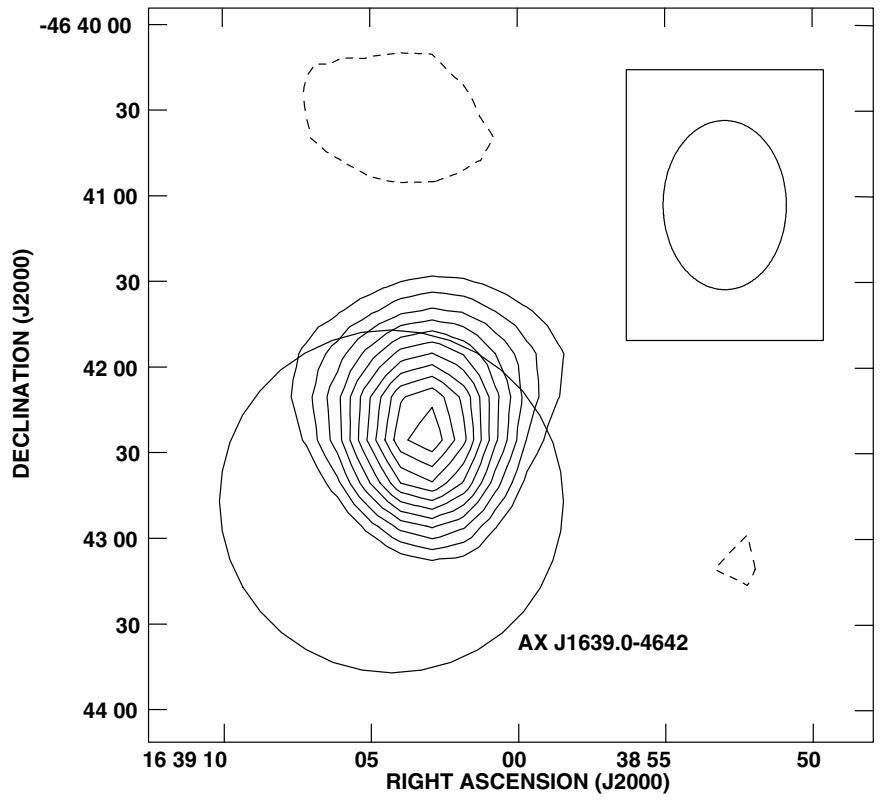

Fig. 3. Contour image of the MGPS data obtained with MOST at $843 \mathrm{MHz}$ on 1992 April 16. The image size is $4^{\prime} \times 4^{\prime}$. The radio source MOST J1639.0-4642 is well within the $90 \%$ uncertainty error circle of the X-ray source AX J1639.0-4642. Contours are -2, 2, 3, 4, 5, 6, $7,8,9,10,11$ and 12 times the rms noise level of $10 \mathrm{mJy}$. The ellipse in the top right corner is the convolving beam of $59.2 \times 43.0 \mathrm{arcsec}$ in $\mathrm{PA}=0^{\circ}$.

is point-like when the beam size and the presence of nearby sources are considered. We have fitted the images of the two epochs with Gaussian functions and we have obtained peak flux densities of $140 \pm 14$ and $132 \pm 11 \mathrm{mJy} \mathrm{beam}^{-1}$, respectively. The position of the radio source in galactic and equatorial coordinates is $(l, b)=\left(337^{\circ} 995,+0^{\circ} .079\right),(\alpha, \delta)_{\mathrm{J} 2000.0}=$ $\left(16^{\mathrm{h}} 39^{\mathrm{m}} 03^{\mathrm{s}} \cdot 0,-46^{\circ} 42^{\prime} 20^{\prime \prime}\right)$ ( $3 \sigma$ uncertainty of $\left.15^{\prime \prime}\right)$. We note that although the formal errors in right ascension and declination of MGPS are $1^{\prime \prime} \times 1^{\prime \prime} \csc |\delta|$ for sources stronger than $20 \mathrm{mJy}$, sidelobes from nearby strong sources can make these uncertainties much higher. In fact, the peak and the fitted Gaussian center positions at the two epochs change by several arcseconds. Therefore, we have estimated the $1 \sigma$ error radius in position to be $5^{\prime \prime}$.

To search for the radio source at higher frequencies we have inspected the data from the $4.85 \mathrm{GHz}$ PMN single dish survey (Condon et al. 1993). We applied to these data an additional Gaussian filtering process (see Combi et al. 1998 for details) to remove the background diffuse radiation on scales larger than $8^{\prime}$. After several iterations no radio source was found up to a $3 \sigma$ level of $\sim 15 \mathrm{mJy}$.

At near infrared (NIR) wavelengths we have inspected the 2 Micron All Sky Survey (2MASS, Cutri et al. 2003), and found 10 sources in the $3 \sigma$ error circle in position of MOST J1639.0-4642, some of them visible in the $K_{\mathrm{s}}$-band image shown in Fig. 4. At optical wavelengths we have searched in the USNO-B1.0 catalog (Monet et al. 2003), and found 6 sources in the same error circle, of which 3 are located within 1 arcsec of 2MASS sources. 


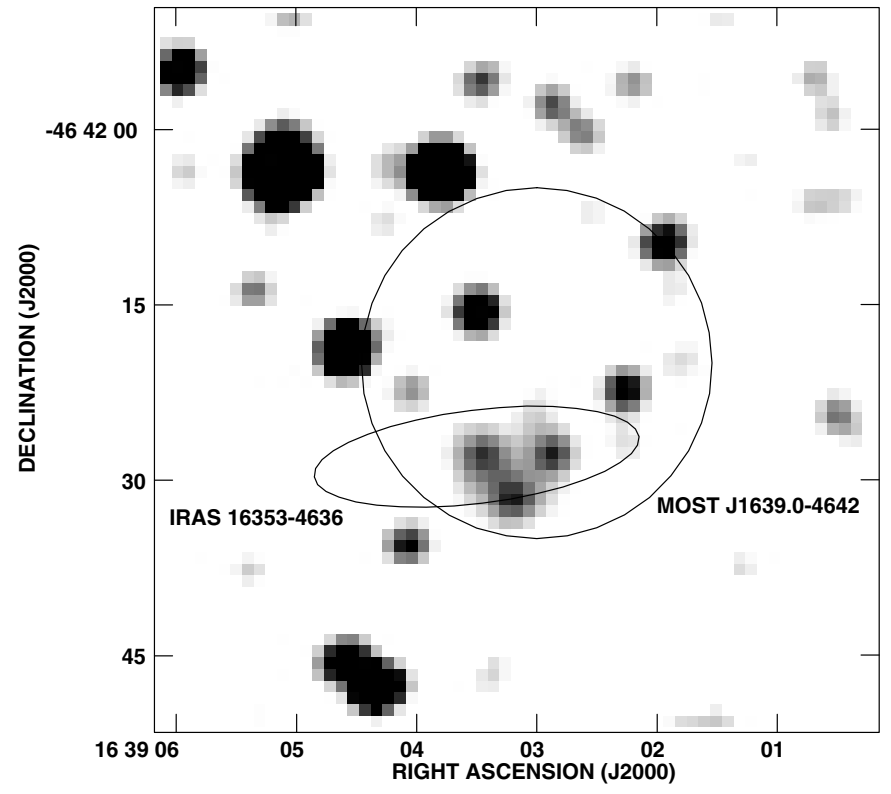

Fig. 4. 2MASS $K_{\mathrm{s}}$-band $60^{\prime \prime} \times 60^{\prime \prime}$ image of the environment of the radio source MOST J1639.0-4642, whose $3 \sigma$ position error circle is shown, together with the $2 \sigma$ position error ellipse of the far infrared source IRAS 16353-4636. Several NIR sources from the 2MASS catalog are possible counterparts of both sources.

At the far infrared part of the spectrum, from 12 to 100 microns, we found that the source IRAS $16353-4636^{1}$ lies inside the error box of the X-ray source. This infrared source is located at $(l, b)=\left(337.995,+0^{\circ} .077\right),(\alpha, \delta)_{\mathrm{J} 2000.0}=$ $\left(16^{\mathrm{h}} 39^{\mathrm{m}} 03^{\mathrm{s}} .5,-46^{\circ} 42^{\prime} 28^{\prime \prime}\right)(95 \%$ or $2 \sigma$ uncertainty ellipse of $14^{\prime \prime} \times 4^{\prime \prime}$ in $\left.\mathrm{PA}=97^{\circ}\right)$. The infrared fluxes at $12,25,60$ and 100 microns are $12.5 \pm 0.6,73.3 \pm 3.7,<806$ ( $3 \sigma$ upper limit) and $2230 \pm 330 \mathrm{Jy}$, respectively. After correction for the slope of the spectrum, these fluxes become $13.5 \pm 0.7,80.5 \pm 4.0$, $<806$ ( $3 \sigma$ upper limit) and $2210 \pm 330 \mathrm{Jy}$, respectively. This source overlaps the southern part of the MOST J1639.0-4642 $3 \sigma$ position error circle, and its uncertainty ellipse in position contains several 2MASS sources, as can be seen in Fig. 4.

The X-ray source AX J1639.0-4642 has been recently re-discovered at higher energies with the IBIS telescope onboard the INTEGRAL satellite, dubbed IGR J16393-4643 (Malizia et al. 2004). It is located at $(l, b)=(338.02,+0.04)$, $(\alpha, \delta)_{\mathrm{J} 2000.0}=\left(16^{\mathrm{h}} 39^{\mathrm{m}} 18^{\mathrm{s}},-46^{\circ} 43^{\prime} 00^{\prime \prime}\right)(90 \%$ or $1.6 \sigma$ uncertainty of $2^{\prime}$ ). We note that although this error circle does not include either the center of AX J1639.0-4642 or the source MOST J1639.0-4642, both sources fall within the $2 \sigma$ error circle of IGR J16393-4643. This source shows an average flux of $F_{\mathrm{X}(20-100 \mathrm{keV})} \simeq 5 \times 10^{-11} \mathrm{erg} \mathrm{cm}^{-2} \mathrm{~s}^{-1}$, and presents a factor of 2-3 flux variability on timescales of months.

Finally, it is interesting to point out that AX J1639.0-4642 lies inside the $95 \%$ location contour of the unidentified $\gamma$-ray source 3EG J1639-4702 (Hartman et al. 1999), as can be seen in Fig. 5. The source is located at $(l, b)=(337.75,-0.15)$, $(\alpha, \delta)_{\mathrm{J} 2000.0}=\left(16^{\mathrm{h}} 39^{\mathrm{m}} 07^{\mathrm{s}},-47^{\circ} 02^{\prime} 24^{\prime \prime}\right)$, and has a $2 \sigma$ radius of 0.56 . Its $\gamma$-ray flux is $(53.2 \pm 8.7) \times 10^{-8}$ photon $\mathrm{cm}^{-2} \mathrm{~s}^{-1}$, and it presents a steep $\gamma$-ray spectral index of $\Gamma=2.5 \pm 0.18$.

\footnotetext{
${ }^{1}$ http://cdsweb.u-strasbg.fr/viz-bin/Cat?II/125
}

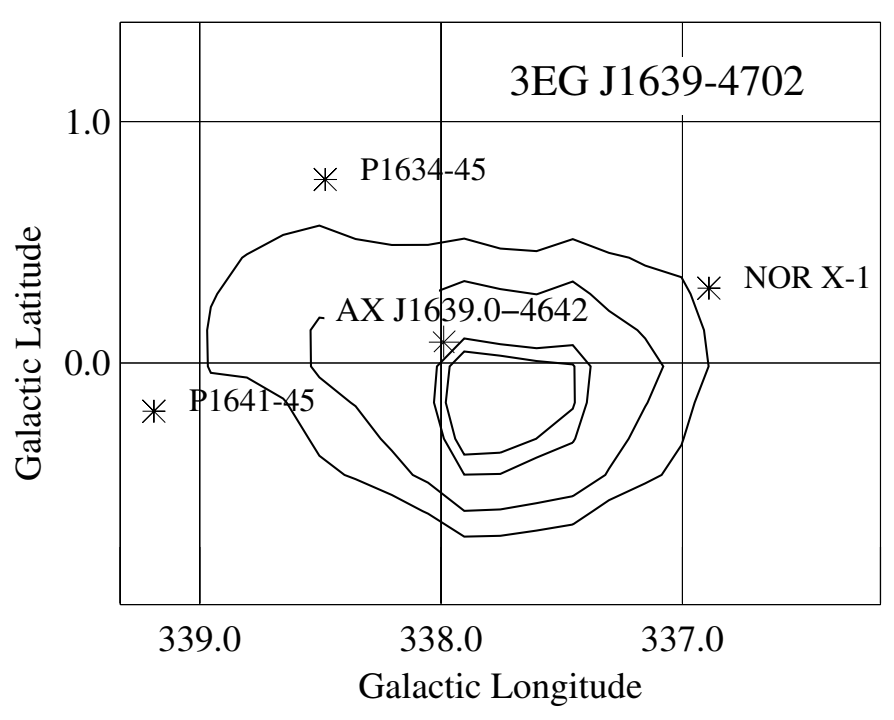

Fig. 5. Gamma-ray probability contours $(50 \%, 68 \%, 95 \%$, and $99 \%$, from inside to outside) of the unidentified source 3EG J1639-4702. The position of the X-ray source AX J1639.0-4642, well within the $95 \%$ probability contour, is marked with an asterisk.

The positions of all these sources are summarized in Table 2.

\section{Discussion}

\subsection{On the nature of $A X J 1639.0-4642$}

At radio wavelengths, the detection at $843 \mathrm{MHz}$ (observations on 1990 June 9 and 1992 April 16) and the non-simultaneous non-detection at $4.85 \mathrm{GHz}$ (observations in 1990 JanuaryMarch), imply that either the source is highly variable on timescales of months or that it has a steep spectral index $\alpha<-1$ (defined in such a way that $S_{v} \propto v^{+\alpha}$ ). In both cases, a non-thermal origin of the radio emission is clearly favored. Therefore, the radio source detected inside the error box of AX J1639.0-4642 could be the manifestation of synchrotron emission from a jet.

The flux variability of AX J1639.0-4642 shown in Fig. 1 and the X-ray spectrum shown in Fig. 2 suggest that it is an HMXB, as already pointed out by Sugizaki et al. (2001). In addition, AX J1639.0-4642 appears to be a persistent X-ray source, since it was detected by ASCA and INTEGRAL, which again supports an HMXB. Finally, the factor of 2-3 flux variability on timescales of months of IGR J16393-4643 also points towards an HMXB.

The obtained value of $N_{\mathrm{H}}$ above $10^{23} \mathrm{~cm}^{-2}$ is reminiscent of the population of persistent and highly absorbed IGR sources detected by INTEGRAL towards the Norma spiral arm, a region of high concentration of atomic and molecular gas and OB stars. All these IGR sources (e.g. IGR J16318-4848, Revnivtsev et al. 2003; IGR J16320-4751, Rodriguez et al. 2003) present rather similar hard X-ray spectra, which are typical of HMXB systems. The most interesting characteristic of these sources is the variable hydrogen column density measured in different observations, a fact that supports intrinsic absorption of the sources. 
Table 2. Galactic and equatorial coordinates and position uncertainties of the sources discussed in the text.

\begin{tabular}{lccccl}
\hline \hline Source name & $l$ & $b$ & $\alpha_{(\mathrm{J} 2000.0)}$ & $\delta_{(\mathrm{J} 2000.0)}$ & Pos. uncertainty \\
\hline AX J1639.0-4642 & $337^{\circ} .993$ & +0.072 & $16^{\mathrm{h}} 39^{\mathrm{m}} 04^{\mathrm{s}} .3$ & $-46^{\circ} 42^{\prime} 47^{\prime \prime}$ & $1^{\prime}(90 \%$ or $1.6 \sigma)$ \\
MOST J1639.0-4642 & $337^{\circ} .995$ & $+0^{\circ} .079$ & $16^{\mathrm{h}} 39^{\mathrm{m}} 03^{\mathrm{s}} .0$ & $-46^{\circ} 42^{\prime} 20^{\prime \prime}$ & $15^{\prime \prime}(99.7 \%$ or $3 \sigma)$ \\
IRAS 16353-4636 & $337^{\circ} .995$ & $+0^{\circ} .077$ & $16^{\mathrm{h}} 39^{\mathrm{m}} 03^{\mathrm{s}} .5$ & $-46^{\circ} 42^{\prime} 28^{\prime \prime}$ & $14^{\prime \prime} \times 4^{\prime \prime}$ in PA $=97^{\circ}(95 \%$ or $2 \sigma)$ \\
IGR J16393-4643 & $338^{\circ} .02$ & $+0^{\circ} .04$ & $16^{\mathrm{h}} 39^{\mathrm{m}} 18^{\mathrm{s}}$. & $-46^{\circ} 43^{\prime} 00^{\prime \prime}$ & $2^{\prime}(90 \%$ or $1.6 \sigma)$ \\
3EG J1639-4702 & $337^{\circ} .75$ & -0.15 & $16^{\mathrm{h}} 39^{\mathrm{m}} 07^{\mathrm{s}}$. & $-47^{\circ} 02^{\prime} 24^{\prime \prime}$ & $0.56(95 \%$ or $2 \sigma)$ \\
\hline
\end{tabular}

In this context, we can roughly estimate the visual extinction $A_{V}$ along the line of sight of AX J1639.0-4642 through its relation to $N_{\mathrm{H}}$ obtained from the X-ray spectrum. Using the formula by Predehl \& Schmitt (1995), $\left[A_{\mathrm{V} / \mathrm{mag}}\right]=$ $0.56\left[N_{\mathrm{H}} / 10^{21} \mathrm{~cm}^{-2}\right]+0.23$, and the $N_{\mathrm{H}}$ values up to $90 \%$ confidence limits from the PL model in Table 1, we obtain extinction values in the range $\sim 20-160 \mathrm{mag}$. Using the PL model with $\Gamma=1, A_{\mathrm{V}}$ ranges between $\sim 120-240 \mathrm{mag}$.

The higher estimated values of $A_{\mathrm{V}}$ could be consistent with the binary system lying inside a molecular cloud, a possibility supported by the presence of the CO molecular cloud located between 8-12 kpc (Fig. 17 in Torres et al. 2003). Alternatively, the binary system could be enshrouded by a dense dust envelope, as has been proposed for the IGR sources discussed above. In any of these cases, we would not expect to detect the NIR/optical counterpart.

However, variable absorption has been measured in IGR J16318-4848 through RXTE and XMM observations on timescales of months (Revnivtsev 2003). Moreover, in a study of the properties of the absorbing and line emitting material in this source, Matt \& Guainazzi (2003) have observed that the iron $\mathrm{K}_{\alpha}$ line varies on timescales as short as $1000 \mathrm{~s}$, implying that the emitting region should have the size of the binary system. This is consistent with a picture in which the absorbing material is due to the stream flowing through the Lagrangian point to form an accretion disk. Although the ASCA data are consistent with a constant X-ray flux below $5 \mathrm{keV}$, the poor statistics below $2-3 \mathrm{keV}$ do not prevent a similar situation from occurring in AX J1639.0-4642. In such a case, the absorbing material would be placed within the binary system and very close to the compact object, and we could expect a low value of the extinction towards the companion star, which could be eventually detected.

Although we do not have spectrophotometric information of a NIR/optical counterpart to derive a distance to the source, assuming that it is located in the Scutum-Crux or in the Norma spiral arms, the galactic longitude of $l=338^{\circ}$ leads to distances in the range between 3 and $13 \mathrm{kpc}$.

All these results show that the source AX J1639.0-4642 (=IGR J16393-4643) clearly deserves further attention. High resolution multifrequency radio observations have recently been conducted to assess the expected non-thermal nature of the emission, to infer physical parameters, to look for eventual extended emission and to provide a better position estimate of the source, to check if it is positionally coincident with IRAS 16353-4636 and to look for a NIR counterpart. Further NIR spectroscopy of an eventual counterpart would make it possible to obtain the spectral type of the optical companion, which would allow us to estimate the distance to the source and the luminosity in the different spectral domains. X-ray and soft $\gamma$-ray observations with currently orbiting satellites will make it possible to obtain a much better position estimate of AX J1639.0-4642 and will constrain the spectral parameters of the source, such as the photon index and specially the hydrogen column density and look for its eventual variability.

\subsection{Microquasars as possible counterparts of unidentified EGRET sources}

As reported in the previous section, AX J1639.0-4642 could be associated with the unidentified high-energy $\gamma$-ray source 3EG J1639-4702. Although Torres et al. (2001b) found three radio pulsars inside the $95 \%$ confidence contour of the $\gamma$-ray source, its possible variability (index $I=$ $1.95)$ and steep photon index $\left(\Gamma_{\gamma}=2.50 \pm 0.18\right)$ do not seem to agree with a pulsar origin. Similarly, these properties would rule out an association with the three SNRs found within the $95 \%$ confidence contour (Torres et al. 2003). Moreover, no identified blazar has been found within the $\gamma$-ray contours. Therefore, we suggest that the microquasar candidate AX J1639.0-4642/MOST J1639.0-4642 (=IGR J16393-4643) is the counterpart of 3EG J1639-4702.

The possibility of MQ being $\gamma$-ray emitters was suggested by Paredes et al. (2000), who proposed the association between the HMXB LS 5039 and the unidentified EGRET source 3EG J1824-1514. In their scenario the $\gamma$-rays are produced by Inverse Compton upscattering of stellar UV photons by the non-thermal relativistic electron population that later on will produce the detected radio emission. On the other hand, the X-ray binary system LS I +61 303 has been associated with the EGRET source 3EG J0241+6103 (Tavani et al. 1996; Kniffen et al. 1997; Strickman et al. 1998; Harrison et al. 2000), and recently Massi et al. (2001, 2004) have revealed its MQ nature. If the MQ nature of AX J1639.0-4642 is confirmed, it could be the third MQ source related to a high-energy gammaray source detected by the EGRET telescope. We quote the basic properties of these three $\gamma$-ray sources in Table 3, together with the properties of the proposed X-ray counterparts. It should be noted that LS 5039 and LS I +61 303, and probably AX J1639.0-4642, have massive optical companions, which provide a stellar UV photon field. On the other hand, the compact object appears to be compatible with a neutron star in the cases of LS 5039 (McSwain et al. 2004) and LS I +61 303 (Hutchings \& Crampton 1981). In addition, it is interesting to 
Table 3. Properties of the three $\gamma$-ray sources and the proposed X-ray/optical/radio counterparts. In the cases of the microquasars LS 5039 and LS I +61 303 the luminosity intervals correspond to intrinsic variability of the sources at the corresponding distances, while in the case of AX J1639.0-4642 they reflect the range of assumed possible distances.

\begin{tabular}{cccccccccc}
\hline \hline$\gamma$-ray source & $\Gamma_{\gamma}{ }^{a}$ & $I^{b}$ & $\begin{array}{c}L_{\gamma(>100 \mathrm{MeV})}{ }^{c} \\
\left(\mathrm{erg} \mathrm{s}^{-1}\right)\end{array}$ & X-ray source & $\begin{array}{c}L_{\mathrm{X}(0.7-10 \mathrm{keV})} \\
\left(\mathrm{erg} \mathrm{s}^{-1}\right)\end{array}$ & $\begin{array}{c}L_{\mathrm{radio}(0.1-100 \mathrm{GHz})} \\
\left(\mathrm{erg} \mathrm{s}^{-1}\right)\end{array}$ & $\begin{array}{c}\text { Spectral } \\
\text { type }\end{array}$ & $\begin{array}{c}P_{\text {orb }} \\
(\mathrm{days})\end{array}$ & $\begin{array}{c}d \\
(\mathrm{kpc})\end{array}$ \\
\hline 3EG J1824-1514 & $2.19 \pm 0.18$ & 3.00 & $3.6 \times 10^{35}$ & LS 5039 & $(0.5-5) \times 10^{34 d}$ & $\sim 1.0 \times 10^{31 e} \mathrm{ON}^{e} .5 \mathrm{~V}((\mathrm{f}))^{f}$ & $4.4^{f}$ & $2.9^{g}$ \\
3EG J0241+6103 & $2.21 \pm 0.07$ & 1.31 & $3.1 \times 10^{35}$ & LS I +61 303 & $(1-6) \times 10^{34 h}$ & $(1-17) \times 10^{31 i}$ & $\mathrm{BOVe}^{j}$ & $26.5^{k}$ & $2.0^{l}$ \\
3EG J1639-4702 & $2.50 \pm 0.18$ & 1.95 & $(3-50) \times 10^{35}$ & AX J1639.0-4642 & $(2-40) \times 10^{34}$ & $(0.8-16) \times 10^{31 m}$ & $?$ & $?$ & $3-13 ?$ \\
\hline
\end{tabular}

${ }^{a}$ Hartman et al. (1999); ${ }^{b}$ Torres et al. (2001a); ${ }^{c}$ computed using the photon fluxes and indices from Hartman et al. (1999); ${ }^{d}$ Reig et al. (2003); ${ }^{e}$ Martí et al. (1998), Ribó et al. (1999); ${ }^{f}$ McSwain et al. (2004); ${ }^{g}$ Ribó et al. (2002b); ${ }^{h}$ Paredes et al. (1997); ${ }^{i}$ computed from the values quoted in Strickman et al. (1998); ${ }^{j}$ Hutchings \& Crampton (1981); ${ }^{k}$ Gregory (2002); ${ }^{l}$ Frail \& Hjellming (1991); ${ }^{m}$ computed assuming $\alpha=-1$.

point out that the luminosities obtained in each spectral domain are very similar in all three sources, specially for the shorter distances to AX J1639.0-4642, giving support to the idea that all of them have similar emission processes.

In this context, Kaufman Bernadó et al. (2002) recently presented a model for galactic $\gamma$-ray emission based on precessing MQs with a jet pointing close to the line of sight (or microblazars).

Depending on the parameters introduced in the model, such MQs can explain sources with $\gamma$-ray luminosities in the range of $10^{34}-10^{38} \mathrm{erg} \mathrm{s}^{-1}$ in the observer's frame, perfectly consistent with the values quoted in Table 3 for all three sources. More recently, Romero et al. (2003) introduced a new mechanism for the generation of high-energy $\gamma$-rays in MQs, based on hadronic interactions occurring outside the coronal X-ray region. The three gamma-ray sources under consideration could be part of this hadronic microblazar population.

Observations with the future missions AGILE and GLAST will confirm or reject the proposed association between AX J1639.0-4642 and the high-energy $\gamma$-ray source 3EG J1639-4702.

\section{Conclusions}

In this paper we have re-analyzed the ASCA data of the X-ray source AX J1639.0-4642 (=IGR J16393-4643) and presented a multiwavelength study of emission in the direction of the source from radio to $\gamma$-rays. The huge hydrogen column density, hard spectral index and flux variability suggest that the source is a dust-enshrouded HMXB system. We have found a non-thermal radio source inside its error circle that could be the manifestation of synchrotron emission from a jet, supporting its microquasar nature. On the other hand, apart from AX J1639.0-4642 there are no other known candidate sources within the 95\% location contours of 3EG J1639-4702 capable of generating the $\mathrm{GeV}$ emission detected by EGRET. All these facts suggest that AX J1639.0-4642 (=IGR J16393-4643) is probably a dust-enshrouded galactic microquasar with highenergy $\gamma$-ray emission.

Acknowledgements. We acknowledge Sylvain Chaty and Gustavo E. Romero for useful discussions, and Paula Benaglia for useful information prior to publication. J.A.C. was supported by CONICET (under grant PIP No. 0430/98). J.A.C. is a researcher of the programme Ramón y Cajal funded by the University of Jaén and Spanish
Ministery of Science and Technology. J.A.C. is very grateful to staff of the Service d'Astrophysique (CEA Saclay) and of Universidad de Jaén, where his research for this project was carried out. M.R. acknowledges support by a Marie Curie Fellowship of the European Community programme Improving Human Potential under contract number HPMF-CT-2002-02053. M.R. also acknowledges partial support by DGI of the Ministerio de Ciencia y Tecnología (Spain) under grant AYA2001-3092, as well as partial support by the European Regional Development Fund (ERDF/FEDER). This research has made use of the NASA's Astrophysics Data System Abstract Service, of the SIMBAD database, operated at CDS, Strasbourg, France, and of the NASA/IPAC Extragalactic Database (NED) which is operated by the Jet Propulsion Laboratory, California Institute of Technology, under contract with the National Aeronautics and Space Administration. The Digitized Sky Survey was produced at the Space Telescope Science Institute under US Government grant NAG W-2166. This publication makes use of data products from the Two Micron All Sky Survey, which is a joint project of the University of Massachusetts and the Infrared Processing and Analysis Center/California Institute of Technology, funded by the National Aeronautics and Space Administration and the National Science Foundation.

\section{References}

Combi, J. A., Romero, G. E., \& Benaglia, P. 1998, A\&A, 333, L91 Condon, J. J., Griffith, M. R., \& Wright, A. E. 1993, AJ, 106, 1095

Cutri, R. M., Skrutskie, M. F., van Dyk, S., et al. 2003, VizieR Online Data Catalog, II/246

(http://cdsweb.u-strasbg.fr/viz-bin/Cat?II/246)

Fender, R. P. 2004, Compact Stellar X-Ray Sources, ed. W. H. G. Lewin \& M. van der Klis (Cambridge University Press), in press [arXiv:astro-ph/0303339]

Fender, R. P., \& Hendry, M. A. 2000, MNRAS, 317, 1

Frail, D. A., \& Hjellming, R. M. 1991, AJ, 101, 2126

Green, A. J., Cram, L. E., Large, M. I., \& Ye, T. 1999, ApJS, 122, 207

Gregory, P. C. 2002, ApJ, 575, 427

Harrison, F. A., Ray, P. S., Leahy, D. A., Waltman, E. B., \& Pooley, G. G. 2000, ApJ, 528, 454

Hartman, R. C., Bertsch, D. L., Bloom, S. D., et al. 1999, ApJS, 123, 79

Hutchings, J. B., \& Crampton, D. 1981, PASP, 93, 486

Kaufman Bernadó, M. M., Romero, G. E., Mirabel, I. F. 2002, A\&A, 385, L10

Kniffen, D. A., Alberts, W. C. K., Bertsch, D. L., et al. 1997, ApJ, 486, 126

Malizia, A., Bassani, L., Di Cocco, G., et al. 2004, ATel, 227

Martí, J., Paredes, J. M., \& Ribó, M. 1998, A\&A, 338, L71 
Martí, J., Paredes, J. M., Bloom, J. S., et al. 2004, A\&A, 413, 309

Massi, M., Ribó, M., Paredes, J. M., Peracaula, M., \& Estalella, R. 2001, A\&A, 376, 217

Massi, M., Ribó, M., Paredes, J. M., et al. 2004, A\&A, 414, L1

Matt, G., \& Guainazzi, M. 2003, MNRAS, 341, L13

McSwain, M. V., Gies, D. R., Huang, W., et al. 2004, ApJ, 600, 927

Mirabel, I. F., \& Rodríguez, L. F. 1999, ARA\&A, 37, 409

Monet, D. G., Levine, S. E., Canzian, B., et al. 2003, AJ, 125, 984 (http://cdsweb.u-strasbg.fr/viz-bin/Cat?I/284)

Paredes, J. M., Martí, J., Peracaula, M., \& Ribó, M. 1997, A\&A, 320, L25

Paredes, J. M., Martí, J., Ribó, M., \& Massi, M. 2000, Science, 288, 2340

Paredes, J. M., Ribó, M., \& Martí, J. 2002, A\&A, 394, 193

Predehl, P., \& Schmitt, J. H. M. M. 1995, A\&A, 293, 889

Reig, P., Ribó, M., Paredes, J. M., \& Martí, J. 2003, A\&A, 405, 285

Revnivtsev, M. G. 2003, Astron. Lett., 29, 644

Revnivtsev, M. G., Sazonov, S. Y., Gilfanov, M. R., \& Sunyaev, R. A. 2003, Astron. Lett., 29, 587
Ribó, M. 2003, in ASP Conf. Ser., Future Directions in High Resolution Astronomy: A Celebration of the 10th Anniversary of the VLBA, ed. J. D. Romney \& M. J. Reid

Ribó, M., Reig, P., Martí, J., \& Paredes, J. M. 1999, A\&A, 347, 518

Ribó, M., Ros, E., Paredes, J. M., Massi, M., \& Martí, J. 2002a, A\&A, 394, 983

Ribó, M., Paredes, J. M., Romero, G. E., et al. 2002b, A\&A, 384, 954

Rodriguez, J., Tomsick, J. A., Foschini, L., et al. 2003, A\&A, 407, L41

Romero, G. E., Torres, D. F, Kaufman Bernadó, M. M., \& Mirabel, I. F. 2003, A\&A, 410, L1

Strickman, M. S., Tavani, M., Coe, M. J., et al. 1998, ApJ, 497, 419

Sugizaki, M., Mitsuda, K., Kaneda, H., et al. 2001, ApJS, 134, 77

Tavani, M., Hermsen, W., van Dijk, R., et al. 1996, A\&AS, 120, 243

Torres, D. F., Butt, Y. M., \& Camilo, F. 2001a, ApJ, 560, L155

Torres, D. F., Pessah, M. E., \& Romero, G. E. 2001b, Astron. Nachr., 322, 223

Torres, D. F., Romero, G. E., Dame, T. M., Combi, J. A., \& Butt, Y. M. 2003, Phys. Rep., 382, 303 